\title{
Seronegative spondyloarthritis and Darier's disease: more than a casual association?
}

\author{
C. Cosso', F. Rongioletti ${ }^{2}$, G. Zampogna ${ }^{1}$, D. Camellino', \\ M. Cutolo', M.A Cimmino ${ }^{1}$ \\ ${ }^{1}$ Academic Unit of Clinical Rheumatology and Research Laboratory, \\ Dipartimento di Medicina Interna (DI.M.I.); \\ 2Section of Dermatology, DISSAL, Unity of Pathology, University of Genoa, Genoa, Italy
}

\section{SUMMARY}

A 46-year old man, affected by Darier's disease (DD), was seen because of right hand pain, later extended to shoulders and ankles. Physical examination showed swelling and tenderness of the wrist, metacarpophalangeal and metatarsophalangeal joints, of the right Achilles tendon's enthesis and of the left knee, with psoriatic-like lesions of the scalp. A diagnosis of seronegative spondyloarthritis, supported by HLA-B27 positivity and by the magnetic resonance imaging finding of hand synovitis and unilateral sacro-iliitis, was made. The correlation between DD, spondyloarthritis and psoriasis has been already anecdotally reported. Further observations may clarify if this association is more than casual.

Key words: Seronegative spondyloarthrits, Darier's disease, Arthritis.

Reumatismo, 2013; 65 (2): 86-89

\section{INTRODUCTION}

$\mathrm{D}$ arier's disease (DD), also known as Lutz-Darier-White dyskeratosis follicularis, follicular keratosis or acantholytic dyskeratosis, is an autosomal dominant genodermatosis, reported for the first time independently by Darier and White in 1889 (1). Its prevalence varies between 1:36,000 in England (2) and 1:100,000 in Denmark (3), regardless of gender, but the expression is usually milder in women. Although it is an autosomal dominant disease, approximately $47 \%$ of patients do not report affected relatives (4). The mutated gene is ATP2A2, on chromosome 12q23-24, which encodes SERCA2 (isoform of $\mathrm{Ca}++$ ATPase of the sarcoplasmic reticulum). Anomalies of $\mathrm{Ca}++$ pump can interfere with the growth and the differentiation of cells and with the assembly of desmosomes, with consequent rupture and acantholysis (5-7).

DD is characterized by warty red-brown, extrafollicular and follicular papules and/ or plaques involving largely the seborrheic sites (comprising the scalp, forehead, retroauricular folds, upper arms, front and back of the central trunk, and flexures), palmo- plantar pits, and distinctive nail abnormalities $(1,4)$.

Actually, the therapy of DD is largely unsatisfactory. Traditional treatments are based on antiseptics, topical steroids, topical retinoids and local emollients or keratolytics. Oral retinoids (e.g., acitretin, isotretinoin, etretinate) are the most effective medical treatment (8).

The co-occurrence of DD with various dermatologic and rheumatic conditions has been anecdotally reported in the literature, including psoriasis vulgaris (9), chronic polyarthritis (10), Sjogren's syndrome (11), spondyloarthritis $(12,13)$ and systemic lupus erythematosus (14).

We describe a patient with DD associated with seronegative spondyloarthritis and psoriasis and discuss a possible pathogenetic link between these conditions.

\section{CASE REPORT}

A 46-year old man, affected by DD, has been visited for the onset of right hand and wrist pain, following an injury 8 months before. The histological diagnosis of DD had 
been previously established by skin biopsy on the basis of the presence of cleavage, loss of epidermal adhesions (acantholysis), irregular upward projection of papillae into the cleavage (villi), round cells and grains with eosinophilic dyskeratosis, confined to the granular layer and to the stratum corneum, and hyperkeratosis.

Arthropathy, which was initially attributed to sympathetic dystrophy, did not respond to treatment with intramuscular clodronate. Six months later, it extended to shoulders and ankles, and low back pain appeared.

At first observation, physical examination showed marked swelling and tenderness of the right wrist, of three right metacarpophalangeal joints, of the enthesis of the right Achilles tendon, and of the left 2nd, 4th and 5th metatarsophalangeal joints. The left knee showed mild effusion. The pain visual analogue scale was $70 \mathrm{~mm}$. The disease activity score 28 based on C-reactive protein (DAS28-CRP) was 6.02 and the bath ankylosing spondylitis disease activity index (BASDAI) was 8.3, indicating a high disease activity. Physical examination of the skin showed several brown keratotic papules merging into plaques at the base of the neck, consistent with DD and some erythematous-desquamative plates on the scalp consistent with psoriasis vulgaris. The patient, however, refused a biopsy of the scalp lesion, deemed useful to confirm this diagnosis.

Blood tests showed neutrophilic leukocytosis $[13,300$ white blood cells $(\mathrm{WBC}) / \mu \mathrm{L}]$, elevated platelet count $(506,000$ platelets/ $\mu \mathrm{L}$ ), anaemia (hemoglobin $10.6 \mathrm{~g} / \mathrm{L}$ ), increased ferritin concentration $(831 \mu \mathrm{G} / \mathrm{L})$, erythrocyte sedimentation rate $120 \mathrm{~mm} / \mathrm{h}$ and C-reactive protein $174 \mathrm{mg} / \mathrm{L}$. Immunoglobulin M rheumatoid factor and anti-cyclic citrullinated peptide antibodies were negative, while the histocompatibility antigen HLA-B27 was positive. The urethral swab was positive for Proteus mirabilis. The synovial fluid aspirated from the knee showed a cloudy appearance, absence of crystals, reduced viscosity, $\mathrm{pH} 7$, and $5700 \mathrm{WBC} / \mathrm{mm}^{3}$, of which $95 \%$ were granulocytes.

Radiographs of hands and wrists showed swelling of the proximal interphalangeal

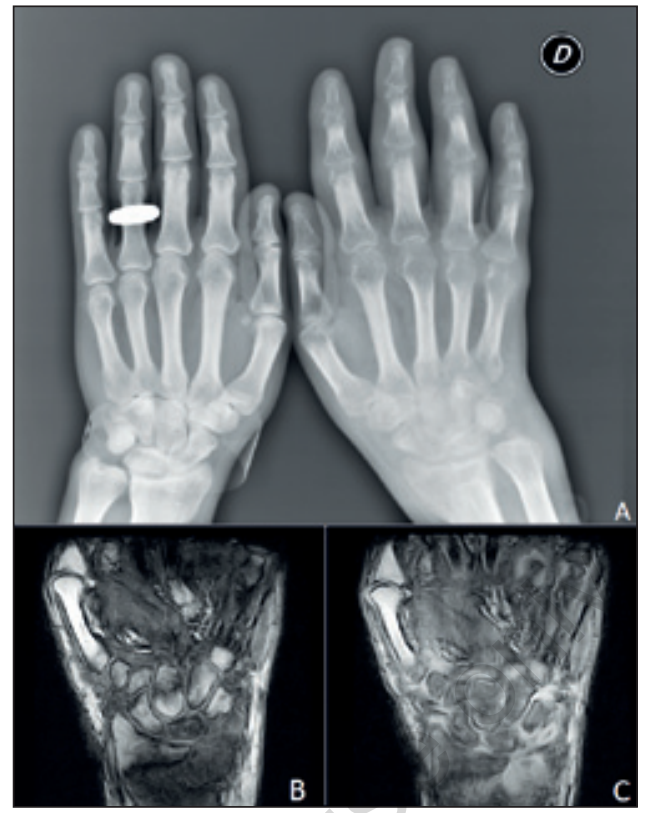

Figure 1 - Anteroposterior radiograph of the hands (A) showing marked soft tissue swelling and iuxta-articular osteoporosis of the right one. Pre- $(\mathrm{B})$ and post-contrast $(\mathrm{C})$ coronal Turbo Spin Echo 3D magnetic resonance imaging sequence of the right wrist: synovial swelling and bone marrow oedema are characterized by contrast enhancement.

joints and marked juxtaarticular osteoporosis (Fig. 1A). Magnetic resonance imaging (MRI) (Fig. 1B and C) showed diffuse bone edema, tenosynovitis and severe synovitis of the wrist, metacarpophalangeal, and interphalangeal joints. MRI of the pelvis showed unilateral sacro-iliitis (Fig. 2A). The enthesis of the Achilles tendon was thickened and inhomogeneous at US, with presence of Doppler signal in the adjoining bursa (Fig. 2B).

A diagnosis of seronegative spondyloarthritis was made and treatment with methotrexate $10 \mathrm{mg} /$ week with folic acid rescue, and prednisone $5 \mathrm{mg} /$ day was started. Fifteen days later, clinic conditions were slightly improved, with DAS28-CRP of 4.83 .

\section{DIscussion}

The correlation between DD and rheumatic conditions has been described in a few 


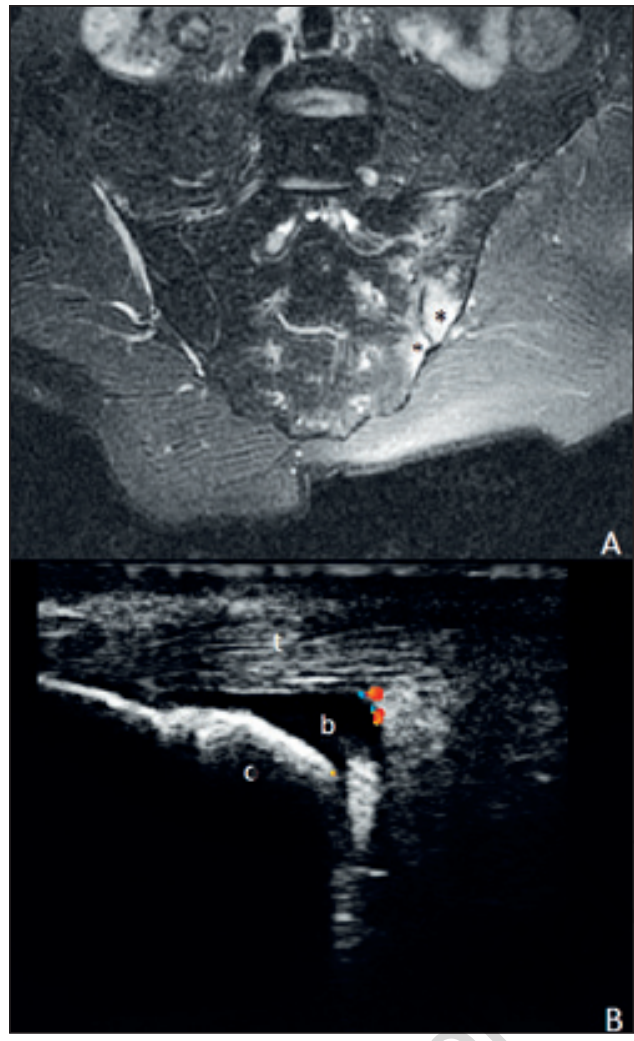

Figure 2 - Short $T$ inversion recovery magnetic resonance imaging sequence of the pelvis (A) shows bone marrow oedema of the left sacro-iliac joint (asterisks). In B an enlarged Achilles tendon (t) is shown at ultrasonography, with concomitant distension of the corresponding bursa (b). A vascular spot is revealed by Doppler mode. c, calcaneum.

anecdotal reports. The most frequent association is seronegative spondyloarthritis $(12,13)$. Psoriasis and DD have also been reported to occur simultaneously (9). Both skin diseases, moreover, are responsive to retinoids, a fact that might have caused underestimation of the incidence of DD.

Our patient presented a clinical picture consistent with the diagnosis of seronegative spondyloarthritis (asymmetric arthritis, enthesitis, sacro-iliitis), confirmed by imaging studies. In addition, the onset of spondyloarthritis after trauma is a relatively frequent event in psoriatic arthritis (15). In conclusion, the correlation between arthritis and DD is not clear. The evaluation of joint involvement in patients with DD is probably not commonly performed, lead- ing to the underestimation of subtle signs, such as minimal arthritis or enthesitis. Joint examinations in DD, as well as the search of clinical manifestations of this genodermatosis in patients with seronegative spondylarthritis, are therefore recommended. Further observations may clarify if the association between DD and seronegative spondylarthritis is more than casual and if psoriasis may be the pathogenetic link between them.

\section{REFERENCES}

1. Macleod RI, Munro CS. The incidence and distribution of oral lesions in patients with Darier's disease. Br Dental J. 1991; 171: 1336.

2. Munro CS. The phenotype of Darier's disease: penetrance and expressivity in adults and children. Br J Dermatol. 1992; 127: 126-30.

3. Svendsen IB, Albrechtsen B. The prevalence of dyskeratosis follicularis (Darier's disease) in Denmark. Acta Derm Venereol (Stockh). 1959; 39: 256-69.

4. Thiagarajan MK, Narasimhan M, Sankarasubramanian A. Darier's disease with oral and esophageal involvement: a case report. Indian J Dent Res. 2011; 22: 843-46.

5. Sakuntabhai A, Burge S, Monk S, Hovnanian A. Spectrum of novel ATP2A2 mutations causes segmental Darier's disease. Hum Mol Genet. 1999; 8: 1611-19.

6. Sakuntabbai A, Dhitavat J, Burge S, Hovnanian A. Mosaicism for ATP2A2 mutations causes segmental Darier's disease. J Invest Dermatol. 2000; 115: 1144-7.

7. MacLennan DH, Brandl CJ, Korczak B, Green NM. Amino-acid sequence of a $\mathrm{Ca} 2++\mathrm{Mg} 2+-$ dependent ATPase from rabbit muscle sarcoplasmic reticulum, deduced from its complementary DNA sequence. Nature. 1985; 316: 696-700.

8. English JC 3rd, Browne J, Halbach DP. Effective treatment of localized Darier's disease with adapalene $0.1 \%$ gel. Cutis. 1999; 63: 227-30.

9. Premalatha S, Yesudian P, Jayakumar S, Thambiah AS. Darier's disease and psoriasis vulgaris. Int J Dermatol. 1981; 20: 52-3.

10. Petera P, Tausch G, Gaismayer K, Horn F. Chronic polyarthritis and erythema anulare centrifugum Darier. Z Rheumatol. 1979; 38: 59-66.

11. Oxholm A, da Cunha Bang F, Oxholm P. Simultaneous occurrence of Darier's disease and primary Sjögren's syndrome. Arthritis Rheum. 1986; 29: 1052. 
12. Saraux A, Bourgeais F, Fauquert P, Le Goff P. Ankylosing spondylitis and Darier's disease. A new spondyloarthropathy? Rev Rhum Mal Osteoartic. 1990; 57: 164-5.

13. Taarit CB, Turki S, Fazaa B, et al. Maladie de Darier et spondylarthrite ankylosante: une nouvelle observation. Rev Med Int. 1997; 18: 821-2.
14. Bassi E, Merlo EM, Scanzi G, et al. Systemic lupus erythematosus and Darier's disease: description of a case. G Ital Dermatol Venereol. 2009; 144: 621-2.

15. Punzi L, Pianon M, Bertazzolo N, et al. Clinical, laboratory and immunogenetic aspects of post-traumatic psoriatic arthritis: a study of 25 patients. Clin Exp Rheumatol. 1998; 16: 277-81. 